\title{
Comparison of different models of orientation selectivity in primary visual cortex
}

\author{
G.E. La Cara \& M. Ursino \\ Department of Electronics, Computer Science, and Systems \\ University of Bologna, Italy.
}

\begin{abstract}
Aim of this work is to compare different models for orientation selectivity in simple cells of the primary visual cortex, in order to gain deeper insight into the role and disposition of cortical connections. Two different models of simple cell response in an hypercolumn are compared. Both receive the same input pattern from the lateral geniculate nucleus, assuming that the geometry of the receptive field can be described through a Gabor function. Moreover, in both models cortical cells receive excitatory synapses from other neurons in the hypercolumn with near orientation preference. The two models differ as to the arrangement of inhibitory connections: in the first, inhibition is in phase opposition with excitation, but with a similar orientation tuning; in the second, inhibition is in phase with excitation, but with larger orientation tuning. Both models, with suitable values of the intracortical synapse parameters, are able to mimic experimental results in the literature. These results include dependence of the response of simple cells on the orientation, contrast and spatial frequency of input gratings or bars. Moreover, both models can reproduce the so-called "cross orientation inhibition": addition of a grating with different orientation significantly reduces the response to an optimally oriented grating. These results demonstrate that different models of intracortical connectivity in the primary visual cortex are compatible with experimental data.
\end{abstract}

\section{Introduction}

The main characteristic of simple cells in the primary visual cortex is orientation selectivity. As originally discovered by Hubel and Wiesel in their pivotal works at the early sixties, these cells respond preferentially to elongated visual stimuli of a particular orientation and a particular spatial frequency [1]. 
There is much consensus today that orientation selectivity in cortical simple cells arises from the combination of a feedforward mechanism from the lateral geniculate nucleus to the primary visual cortex, and a feedback mechanism involving intracortical connections. The feedforward mechanism, in particular, should be responsible to set an initial orientation bias for the response of simple cells, while intracortical connections should amplify the response and determine some of the observed experimental properties, such as contrast invariance and suppression of non-optimal stimuli.

Although the presence of intracortical excitatory and inhibitory synapses is well documented in recent physiological studies [2], their exact role and reciprocal importance in affecting the response of simple cells is still controversial. Especially, the exact arrangement and function of the intracortical circuitry responsible for inhibition requires additional study.

In recent years two main theories on orientation selectivity of cortical visual cells have been proposed. In the push-pull model by Troyer et al. [3], inhibition comes from interneurons with a receptive field anticorrelated with the receptive field of the target simple cells: as a consequence, inhibition is always in spatial phase opposition with excitation. In the emergent model by Somers et al. [4] inhibition comes form interneurons whose receptive fields have the same spatial phase as target cortical cells, but the inhibitory synapses have a broader spatial extension than the excitatory ones.

Both models are quite complex, including integrate-and-fire neurons, thousand of synapses and massive physiological details. As a consequence, these models are computationally onerous, the analysis of their results is often not intuitive, and they cannot be easily integrated within larger models of the visual elaboration system.

Aim of this work is to present simpler models of orientation selectivity in the visual cortex, which maintain the main functional characteristics of the original computational models, but with a much simpler mathematical structure. The two models are then used to analyze whether the hypotheses on intracortical circuitry are able to explain physiological results, and to look for experiments able to look for the more realistic patterns of intracortical connectivity.

\section{Model description}

In the present model the output of neurons is not represented as a series of action potentials (spikes) but as a continuous quantity describing the firing rate. Moreover, the model considers only the architecture of a single hypercolumn. We have 180 excitatory neurons and 45 inhibitory interneurons. The neurons are parameterized by their preferred orientation. Since in this model we do not consider movement and direction selectivity, the orientation angle is restricted to the range $90^{\circ}-270^{\circ}\left(180^{\circ}\right.$ representing the horizontal direction, $90^{\circ}$ and $270^{\circ}$ the vertical direction).

The model comprehends three different elaboration steps:

i) a description of the receptive fields of thalamic cells, including both ONcenter and OFF-center cells. Receptive fields of these cells have been described 
as the difference between gaussian functions, having the same spatial constant in both directions. Hence, the following function holds for a thalamic cell at position $x, y:$

$$
\varphi_{x, y}(i, j)=A_{1} \cdot e^{-\frac{(i-x)^{2}+(j-y)^{2}}{r_{1}^{2}}}-A_{2} \cdot e^{-\frac{(i-x)^{2}+(j-y)^{2}}{r_{2}^{2}}}
$$

where $x$ and $y$ are the coordinates for the centre of the thalamic cell, $r_{1}$ and $r_{2}$ are the characteristic radii of the central and surround regions, and $A_{1}$ and $A_{2}$ are the peak sensitivities in the center and surround.

The output activity of the thalamic cell is computed, starting from the knowledge of its receptive field and input luminance, by considering the presence of two nonlinear effects. i) the activity cannot decrease below zero; ii) the thalamic output to sinusoidal gratings increases with contrast, but exhibits progressive saturation if contrast approaches $0.3-0.35[5]$.

ii) a description of the receptive fields of simple cells in the cortex. The receptive fields are constructed using afferent inputs from 15 thalamic cells, oriented along the preferred orientation of the cell and sampled by means of a Gabor function (see figure 1 left).
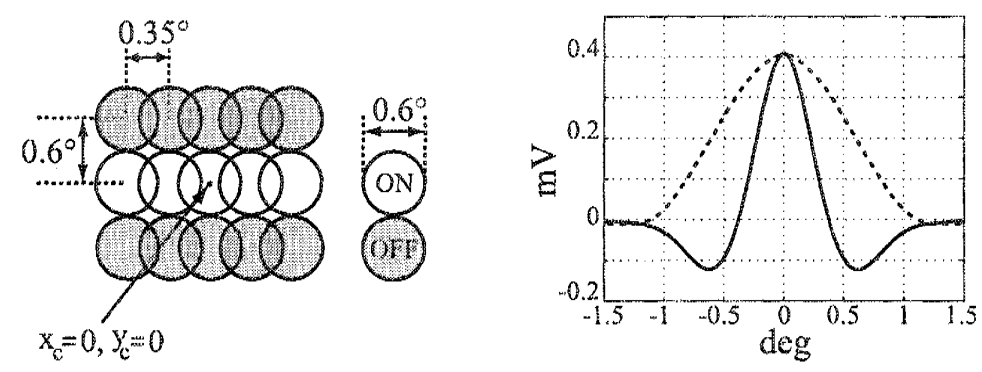

Figure 1: Left panel: thalamic input to a simple cell, arising from 15 thalamic cells arranged in a regular lattice. Right panel: two orthogonal sections of the receptive field along the central vertical direction $\left(x_{c}=0\right.$, continuous line) and along the central horizontal direction $\left(y_{c}=0\right.$, dashed line).

The value of the synapses from thalamic cells to the cortical cell with orientation preference $\vartheta$ obey to the following law:

$$
\begin{aligned}
& W_{c t}\left(x_{g}-x_{c}, y_{g}-y_{c}\right)= \\
& =W_{c t 0} \cdot e^{-\left[\frac{\left(u_{g}-x_{c}\right)^{2}}{\sigma_{x}^{2}}\right]} \cdot e^{-\left[\frac{\left(v_{g}-y_{c}\right)^{2}}{\sigma_{y}^{2}}\right]} \cdot \cos \left(2 \pi f\left(y_{g}-y_{c}\right)+\varphi\right)
\end{aligned}
$$

where $\left(x_{c}, y_{c}\right)$ are the coordinates of the centre of the cortical cell receptive field, $\left(x_{g}, y_{g}\right)$ are the coordinates of the centre of the receptive field for the geniculate 
cell $(g=1,2, \ldots 15)$ and $\vartheta$ is a rotation angle, describing the orientation preference of the simple cell. The variables $u_{g}$ and $v_{g}$ describe a rotation of the plane, i.e.,

$$
u_{g}=x_{g} \cos (\vartheta)+y_{g} \sin (\vartheta) \quad v_{g}=-x_{g} \sin (\vartheta)+y_{g} \cos (\vartheta)
$$

Parameters $\sigma_{x}^{2}$ and $\sigma_{y}^{2}$ in eqn (2) set the dimension of the receptive field. The spatial frequency, $f$, determines the width of the $\mathrm{ON}$ and OFF subregions, and the phase $\varphi$ allows simulation of both $\operatorname{ON}(\varphi=0)$ and $\operatorname{OFF}(\varphi=\vartheta)$ cortical cells. The values of parameters $\sigma_{x}^{2}, \sigma_{y}^{2}$, and $f$ have been chosen to obtain a receptive field in agreement with physiological data $[6,7]$. Two orthogonal sections of the receptive field, obtained from eqns (1), (3) by moving a spot of light along the central vertical and horizontal directions, are illustrated at the right of figure 1 .

iii) the presence of excitatory and inhibitory connections between cortical cells. In fact, cortical neurons do not only receive input from thalamic cells (as described above) but also excitatory synapses from the other cortical cells in the hypercolumn, and inhibitory synapses from additional inhibitory interneurons. In order to describe the cortical circuitry, the following equations have been used

$$
\begin{gathered}
\Delta V_{c}^{O N}(\vartheta, t)=\Delta V_{c t}^{O N}(\vartheta)+\Delta V_{c e}^{O N}(\vartheta, t)-\Delta V_{c i}^{O N}(\vartheta, t) \\
\tau \frac{d c^{O N}(\vartheta, t)}{d t}=-c^{O N}(\vartheta, t)+k_{c}\left[\Delta V^{O N}(\vartheta, t)-\vartheta\right]^{+}
\end{gathered}
$$

where $V(\vartheta, t)$ is the membrane potential of the cortical cell with orientation preference $\vartheta$ at time $t, c(\vartheta, t)$ is the output activity of the cortical cell at time $t, \Delta V_{c t}(\vartheta)$ are the changes in membrane potential caused by the geniculate input, $\Delta V_{c e}(\vartheta, t)$ and $\Delta V_{c i}(\vartheta, t)$ are the changes in membrane potential caused by excitatory and inhibitory intracortical connections, respectively. eqn (4) simply signifies that the overall change in membrane potential is the sum of contributions from thalamic and cortical (excitatory and inhibitory) inputs. eqn (5) summarizes the dynamics of the cortical cells response by means of a first-order differential equation with time constant $\tau$. At equilibrium, the value of cortical cell activity is obtained by comparing the variation in membrane potential with a threshold, $v$, using a single wave rectifier [ ${ }^{+}$which cuts negative values, and multiplying the value so obtained by a gain factor $k_{c}$.

In this work two different models are compared. These differ as to the arrangement of inhibitory synapses: in the first model, inhibition is in spatial phase opposition with excitation; in the second, inhibition is in phase with excitation. The first model is realized assuming that inhibitory interneurons directed to ON cortical cells have an OFF type receptive field form the geniculate inputs (i.e., we used $\varphi=\pi$ in eqn (2) for the inhibitory interneurons, and $\varphi=0$ for the cortical cells). The second model is realized assuming that inhibitory interneurons directed to ON cells have an $\mathrm{ON}$ receptive field (i.e., $\varphi=0$ in both cells). The two models are depicted in figure 2 .

Moreover, according to physiological data [8] both models assume that the synaptic strength between cortical cells, and between cortical cells and inhibitory interneurons decreases with the distance in orientation preference. This means that 
a)

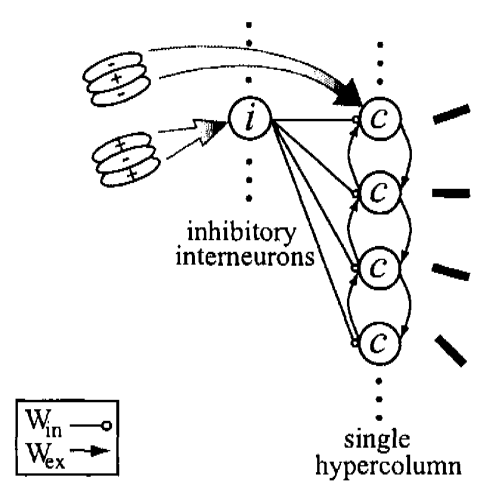

b)

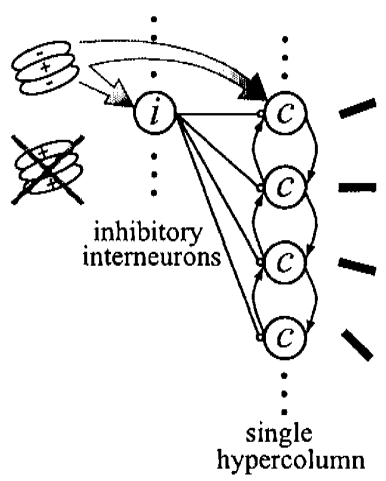

Figure 2: The arrangement of thalamic excitatory, cortical excitatory and cortical inhibitory inputs to simple cells according to the two models.

two neurons with similar orientation preference have a strong synaptic connection, whereas neurons with a large difference in orientation preference are connected by means of weaker synapses. Accordingly, the following equations have been used to compute the excitatory and inhibitory cortical inputs to a cortical cell with preferred orientation $\vartheta$.

$$
\begin{gathered}
\Delta V_{c e}^{O N}(\vartheta, t)=\sum_{\varphi} w_{e x}(\vartheta-\varphi) c^{O N}(\varphi, t) \\
\Delta V_{c i}^{O N}(\vartheta, t)=\sum_{\varphi} w_{i n}(\vartheta-\varphi) i^{O F F / O N}(\varphi)
\end{gathered}
$$

where the symbols $w_{e x}(\vartheta-\varphi)$ and $w_{\text {in }}(\vartheta-\varphi)$ represent the excitatory synapses from a simple cell with orientation $\varphi$ to a simple cell with orientation $\vartheta$ and the same spatial phase (ON vs. $\mathrm{ON}$ ), and $w_{\text {in }}(\vartheta-\varphi)$ represents the synapses from an inhibitory interneuron (orientation preference $\varphi$ ) to a simple cell (orientation preference $\vartheta$ ). As specified above, the inhibitory interneuron has opponent spatial phase (OFF) in the first model, but the same spatial phase (ON) in the second one.

Dependence of the synaptic strength of the distance in orientation preference has been implemented using a Gaussian relationship. Hence, we have

$$
\begin{aligned}
& w_{e x}(\vartheta-\varphi)=a \cdot e^{-b\left[\delta(\vartheta-\varphi)^{2}\right]} \\
& w_{i n}(\vartheta-\varphi)=c \cdot e^{-d\left[\delta(\vartheta-\varphi)^{2}\right]}
\end{aligned}
$$


Table 1: parameters of the model

\begin{tabular}{|l|c|c|c|c|}
\hline & $a$ & $b$ & $c$ & $d$ \\
\hline Model I & $0.007 \mathrm{mV} /($ spikes $/ \mathrm{sec})$ & 100 & $0.2 \mathrm{mV} /($ spikes $/ \mathrm{sec})$ & 210 \\
\hline Model 2 & $0.018 \mathrm{mV} /($ spikes $/ \mathrm{sec})$ & 140 & $0.012 \mathrm{mV} /($ spikes $/$ sec $)$ & 1.5 \\
\hline
\end{tabular}

where the "orientation distance", $\delta$, is computed as follows

$$
\delta(\vartheta-\varphi)= \begin{cases}\frac{|\vartheta-\varphi|}{90^{\circ}} & \text { if } 1^{\circ} \leq|\vartheta-\varphi| \leq 90^{\circ} \\ \frac{\left[180^{\circ}-|\vartheta-\varphi|\right]}{90^{\circ}} & \text { if } 90^{\circ} \leq|\vartheta-\varphi| \leq 180^{\circ}\end{cases}
$$

where $a, b, c$ and $d$ are constant parameters, and $\delta(\vartheta-\varphi)$ represents the distance between the preferred orientations, normalized between 0 (equal orientation), and $1\left(\right.$ maximal orientation difference $\left.=90^{\circ}\right)$.

\section{Results}

The two mathematical models were implemented on Pentium-based personal computers, using the software package Matlab 6.1.

The models have been stimulated by means of gratings and bars with different orientation, contrast and spatial frequency.

Various experimental evidences on the response of simple cortical cells, reported in the physiological literature, were simulated with each model. A first group of experimental data was used to assign intracortical synapses. The others data provided test for model validation.

Assignment of cortical synapses: the contrast invariance of the orientation tuning curve - Experimental data show that cortical simple cells are tuned for orientation of the input grating. The orientation tuning curve exhibits an half-width at half-height $(\mathrm{HWHH})$ in the range $20^{\circ}-25^{\circ}[9,10]$. Moreover, this orientation selectivity is independent of the contrast of the input stimulus. Stimuli of higher contrast evoke a greater peak response, but do not significantly modify the HWHH. Both models, with suitable values of intracortical synaptic weights, are able to simulate this behavior quite well, as shown in the two panels of figure 3. In order to achieve this behavior, however, parameters describing the distribution of intracortical excitatory and inhibitory synapses (i.e, parameters $a, b, c$ and $d$ in eqns (8), (9)) must be assigned different values for the two models (see table 1). In particular, in the first model excitatory synapses are weaker than the inhibitory ones, but the two groups of synapses have quite similar orientation tuning. By contrast, in the second model, excitatory synapses are stronger than the inhibitory ones, but exhibit a 
a)

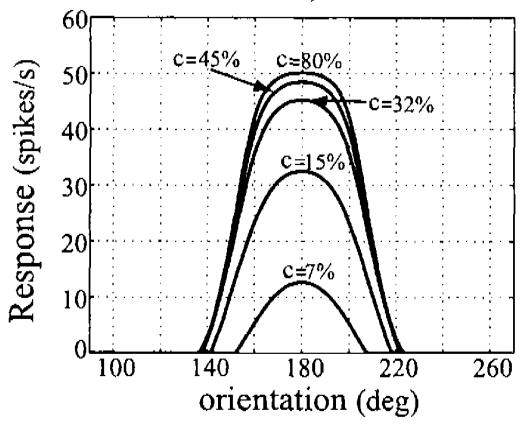

b)

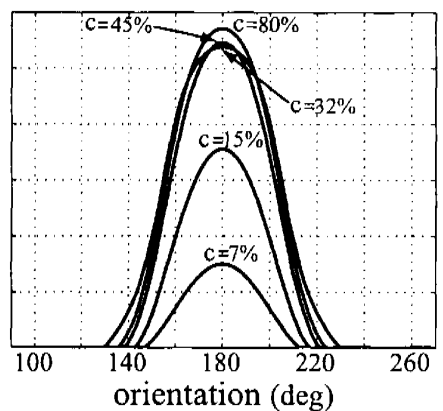

Figure 3: Activity of simple cells vs. orientation (i.e., the orientation curve) at different values of contrast for the two models. This figure can be equally interpreted as the response of one cell (preferred orientation $=180^{\circ}$ ) to input gratings with different orientations, or the response of all 180 cells in the hypercolumn to a single grating.

very sharp orientation tuning, whereas inhibitory synapses are only poorly tuned for orientation.

Model validation - Both models, with assigned parameter values, were then used to simulate additional features of the simple cortical cells. Two well-known properties of simple cell response will be tested below:

i) The HWHH of the orientation curve decreases when the spatial frequency of the input grating is increased $[11,12,13]$. Both models verify this property, in acceptable agreement with physiological data (figure 4).

a)

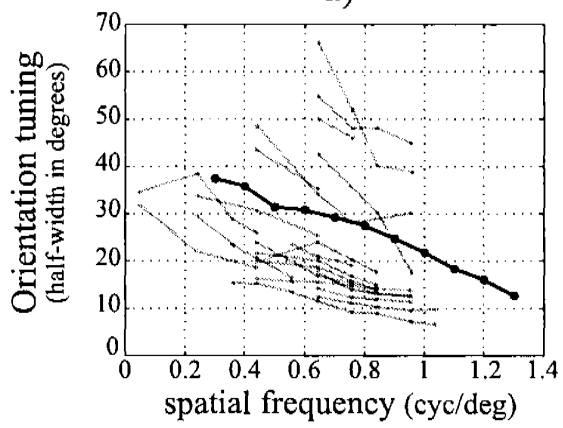

b)

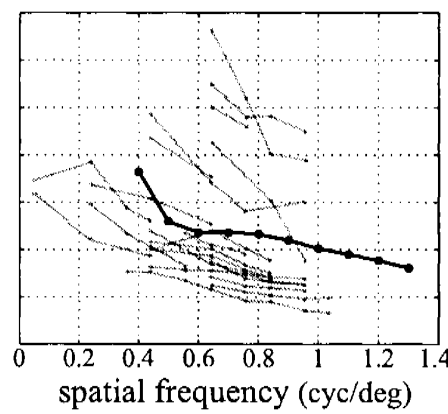

Figure 4: Half width at half height of the orientation curve, computed with the two models at different values of spatial frequency (black thick line). By way of comparison, experimental points from [11] are also reported (gray curves). 
ii) The response of a simple cell to two superimposed gratings with different orientation is significantly lower than the sum of the individual responses, even if the second grating would evoke negligible response on the cell if applied alone [14]. This phenomenon, named "suppression by non optimal stimuli" is illustrated in figure 5, where two gratings with identical contrast, but separated by an angle $\pm 60^{\circ}$, are applied to the model, first separately, then in combination. In both models, a cell which responds optimally to the first grating but with a negligible behavior to the second, exhibits a significant reduction in the peak response (from 40-50 spikes/s to about 10 spikes/s) when the two stimuli are applied simultaneously.

a)
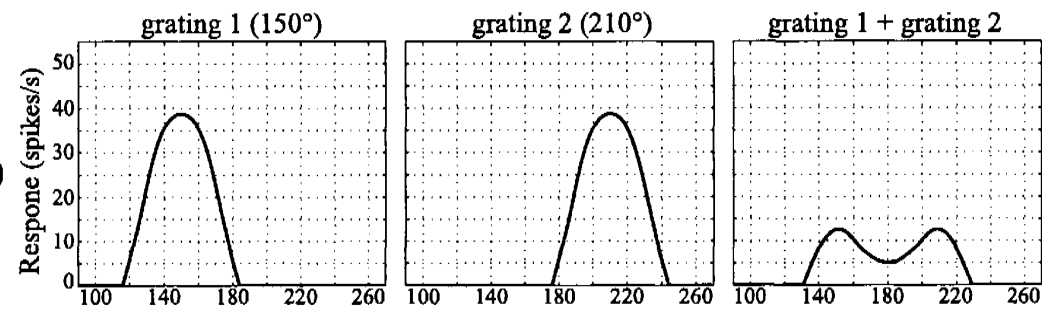

b)
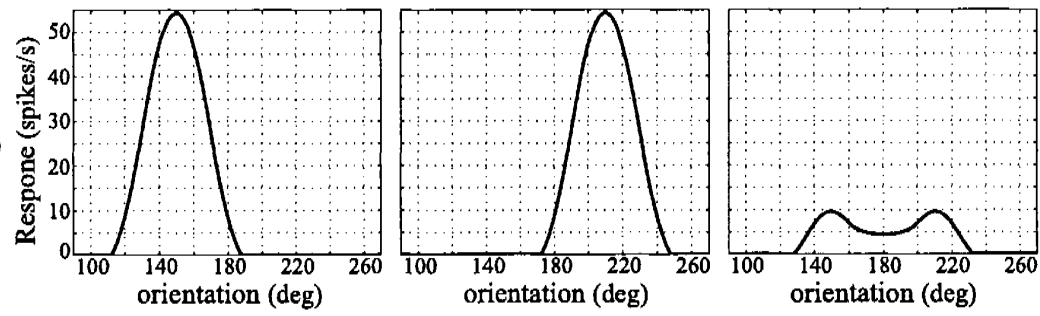

Figure 5: Effect of a second grating with non-optimal orientation superimposed on a first grating in the first model (upper panels) and the second model (lower panels). The left and middle panels represent the effect of two gratings, separated by an angle $60^{\circ}$, applied separately. The right panel represents the effect of the two gratings applied simultaneously. The abscissa has the same meaning as in figure 3 .

\section{Discussion}

A first objective of the present study was to realize models of orientation selectivity in cortical simple cells, which represent a good compromise between physiological reliability and mathematical simplicity. In fact, the proposed equations can be simulated on personal computers quite easily but, at the same time, they include various important physiological details, such as the structure of the receptive fields of thalamic and simple cortical cells, and reliable architectures for the intracortical circuitry. 
A second objective of the study was to test whether different mechanisms of intracortical inhibition can be compatible with experimental data. As clearly discussed by Ferster and Miller in their recent review paper [15], in fact, intracortical inhibition is essential to explain several well-known properties of the simple cell response, such as contrast invariance of the orientation tuning and inhibition by non-optimal stimuli. However, despite the great number of experimental studies appeared on this subject in the last decades, the structure of intracortical inhibition in simple cells of the visual cortex is still a matter of debate.

The present simulation results demonstrate that different architectures for intracortical inhibition are able to explain experimental evidences quite well, provided suitable values are assigned to intracortical synapse parameters. In both models, excitatory and inhibitory intracortical synapses depend on the distance of orientation preference between the presynaptic and postsynaptic cells. This assumption agrees with experimental observations on intracortical circuitry [2]. In the first model, however, inhibition is spatially in phase opposition with excitation, i.e., the receptive field of an inhibitory interneuron responds to dark stimuli where the target cell responds to light stimuli, and viceversa. As hypothesized by Troyer et al. [3], this "push-pull" arrangement can insure contrast invariance, provided inhibition is stronger than excitation and the two cortical connections (inhibitory and excitatory) have similar orientation tuning (see Tab. 1). The second model, originally proposed by Somers et al. [4], assumes that inhibition has the same spatial phase as excitation, i.e., the receptive field of inhibitory interneurons responds to light or dark stimuli in the same way as their target cells. This arrangement of cortical synapses can explain experimental data equally well, if inhibition is weaker than excitation but with a wider orientation tuning. Such particular disposition realizes the classical "mexican hat" function for cortical synapses, typical of many neural network models.

Simulations performed using various gratings and bars, with different contrast, orientation and spatial frequency, as well as using multiple gratings ("plaids") show that the two models are almost indistinguishable on the basis of simple cell activity. Both are able to explain contrast invariance, dependence of output activity on spatial frequency and suppression by non-optimal stimuli.

In conclusion, the choice of the more reliable arrangement of intracortical inhibition requires additional experimental knowledge, especially in regard to the structure of intracortical synapses within the orientation column. Further model improvements may be concerned with the connections among different hypercolumns (i.e., cells which respond to the same orientation but with a receptive field located at different points in the retinal layer) and/or the dynamical response of thalamic and cortical cells to direction and velocity.

\section{References}

[1] Wiesel T. N. Hubel D. Receptive fields, binocular interaction and functional architecture in the cat's visual cortex. J. Physiol., 160, pp. 106-154, 1962.

[2] Shapley R. M. Hawken M. J. Ringach D. L., Bredfeldt C. E. Suppression of 
neural responses to nonoptimal stimuli correlates with tuning selectivity in macaque V1. J. Neurophysiol., 87, pp. 1018-1027, 2002.

[3] Priebe N. J. Miller K. D. Troyer T. W., Krukowski A. E. Contrast-invariant orientation tuning in cat visual cortex:thalamocortical input tuning and correlation-based intracortical connectivity. J. Neurosci., 18, pp. 5908-5927, 1998.

[4] Smith E. L. Somers D. C., Nelson S. B. An emergent model of orientation selectivity in cat visual cortical simple cells. J. Neurosci., 15, pp. 5448-5465, 1995.

[5] Smith E. L. Hamamoto J. Yoshida K. Cheng H., Chino Y. M. Transfer characteristics of lateral geniculate nucleus $X$ neurons in the cat: effects of spatial frequency and contrast. J. Neurophysiol., 74, pp. 2548-2557, 1995.

[6] Palmer L. A. Jones J. P. An evaluation of the two-dimensional gabor filter model of simple receptive fields in cat striate cortex. J. Neurophysiol., 58, pp. 1233-1258, 1987.

[7] Wheat H. Ferster D., Chung S. Orientation selectivity of thalamic input to simple cells of cat visual cortex. Nature, 380, pp. 249-252, 1996.

[8] Ferster D. Orientation selectivity of synaptic potentials in neurons of cat primary visual cortex. J. Neurosci., 6, pp. 1284-1301, 1986.

[9] Albus K. Heggelund P. Orientation selectivity of single cells in striate cortex of cat: the shape of orientation tuning curves. Vision. Res., 18, pp. 1067-1071, 1978.

[10] Freeman R. D. Sclar G. Orientation selectivity in the cat's striate cortex is invariant with stimulus contrast. Exp. Brain. Res., 46, pp. 457-461, 1982.

[11] Siguenza J. A. Vidyasagar T. R. Relationship between orientation tuning and spatial frequency in neurones of cat area 17. Exp. Brain Res., 57, pp. 628$631,1985$.

[12] Palmer L. A. Jones J. P., Stepnoski A. Two-dimensional spectral structure of simple receptive fields in cat striate cortex. J. Neurophysiol., 58, pp. 1212$1232,1987$.

[13] Pomfrett C. J. Hammond P. Influence of spatial frequency on tuning and bias for orientation and direction in the cat's striate cortex. Vision. Res., 30, pp. 359-369, 1990.

[14] Bonds A. B. Role of inhibition in the specification of orientation selectivity of cells in the cat striate cortex. Vis. Neurosci., 2, pp. 41-55, 1989.

[15] Miller K. D. Ferster D. Neural mechanisms of orientation selectivity in the visual cortex. Annu. Rev. Neurosci., 23, pp. 441-471, 2000. 